\title{
Algoritmos Avanzados de Adquisición y Procesamiento de Nubes de Puntos 3D para el Descubrimiento de Información en Mamíferos Marinos
}

\section{Advanced adquisition and processing algorithms for 3D point clouds pertaining to marine mammal information discovery}

\section{Doctorando:}

\section{Almonacid Jonathan Samuel}

Centro para el Estudio de Sistemas Marinos (CESIMAR), Grupo de Estudio de Mamíferos Marinos (GEMM), Laboratorio de Ciencia de las Imágenes Bahía Blanca (LCI), Universidad Tecnológica Nacional - Argentina almonacid@cenpat-conicet.gob.ar

\section{Director/es:}

\section{Delrieux Claudio Augusto}

\section{Co-director/es:}

\section{Lewis Mirtha Noemí}

\section{Resumen}

El Procesamiento de imágenes es una de las ramas de la informática de mayor crecimiento en los últimos tiempos. Esto no solo se debe a la amplitud de sus aplicaciones, sino también a que el avance de la tecnología que se viene experimentando en estos años, junto con el surgimiento de herramientas para un rápido aprovechamiento de las mismas, ha hecho posible que algoritmos cuyos costos de procesamiento eran muy altos hasta hace unos años atrás, puedan ahora ser ejecutados en períodos de tiempo relativamente cortos.

Por otro lado, el abaratamiento de costos en elementos relacionados a la captura de información visual ha incentivado enormemente la realización de estudios afines a su aplicación fuera del ámbito industrial. En este contexto, los sensores de profundidad han abierto un nuevo campo de estudio permitiendo generar y trabajar con modelos tridimensionales de elementos del mundo real tal y como son en lugar de los modelos prediseñados.

En el caso particular de la biología, se ha abierto una posibilidad de mejorar la manera en que los científicos obtienen información relevante a sus investigaciones, siendo este el caso del estudio de mamíferos marinos, más concretamente el Elefante Marino del Sur (Mirounga leonina).

En particular este trabajo se enfoca en la búsqueda de algoritmos apropiados para la limpieza y análisis automatizado de modelos tridimensionales (nubes de puntos) de elefantes marinos. Dicho estudio brindaría a los científicos un método no invasivo para obtener medidas mucho mas rápidas y precisas que las proporcionadas por los métodos utilizados hasta el momento, a la vez que reduciría de manera considerable los riesgos (tanto para los científicos como para los animales) que estos conllevan.

Palabras clave: Nube de Puntos, Procesamiento de imágenes, Modelo 3D.

\footnotetext{
Abstract

In recent years, image processing has undergone great improvements within Computer Science. In part this has been due to the benefits that can derived from its aplications, but a larger part of this development has been because of the improvements in technology and the rise in better suited deployment tools. This has enabled a faster and easier implementation of algorithms which would have been almost imposible to fulfill in the previous decade, because of their process reuirements alone.

Furthermore, the cost reduction of advanced visual recording devices has contriuted enormously to the upsurge in research dedicated to their aplications outside of solely commercial use. Within this context, depth sensors (such as 3D sensors) have opened a whole new research area that has made it more accesible at a
} 
mainstream level, allowing for the creation and use of 3D models that are actually suited to real world objects instead of predesigned ones. This has paved the way for enormous improvements in other areas of discipline such as biology, where the way scientists obtain information regarding their physical environment has been revolutionized.

This work is aimed towards the research of algorithms for automatic denoising, and the analysis of threedimensional models (point clouds) of the southern elephant seal(Mirounga leonina). This investigation wold provide a faster, more accurate and non-invasive method for morphometric data retrieval. This would also greatly reduce the risks and dangers (to both the research teams and the research subjects) that are associated with the more traditional methodologies that are currently used.

Keywords: Point Cloud, Digital image processing , 3D model.

\section{Introducción}

En la actualidad, hay gran cantidad de estudios en animales en los que es necesaria la obtención de medidas referentes al tamaño del mismo. Cuando los animales en cuestión son de dimensiones considerables, la tarea de obtener estas mediciones puede tornarse no solo complicada, sino también arriesgada.

En el estudio de poblaciones de mamíferos tales como el Elefante Marino del Sur (Mirounga leonina), donde los especímenes adultos pueden superar ampliamente las 2 toneladas de peso y medir hasta 5 metros de largo, la tarea de pesar estos animales ha sido dejada de lado en favor de medidas indirectas. En la actualidad, el peso de los animales es derivado a partir del volumen del mismo, el cual es a su vez calculado en base a la medición del largo del animal y algunas medidas de circunferencia en zonas específicas tal como se detalla en Bell (1997).

La tarea simplificada de esta manera aún presenta varios riesgos para los investigadores, los cuales deben sedar al animal para poder tomar las medidas antes mencionadas, para lo cual también se debe destinar tiempo y esfuerzo en el cuidado del individuo después de realizada la tarea.

Este estudio se basa en la utilización de sensores de profundidad de bajo costo, en particular un sensor Structure modelo ST01 (Occipital (2020)), adosado a un dispositivo iPad para la captura de un modelo tridimensional a partir del cual tomar las medidas pertinentes. Dado que el procesamiento de este tipo de datos no es trivial, parte del objetivo que se persigue es lograr que dicho análisis pueda hacerse de manera automatizada, esto es, que los científicos solo tengan que capturar el modelo del animal in-situ, introducirlo al sistema, y poder obtener de manera transparente datos tales como el volumen, peso aproximado y categoría etaria del animal.

En el presente trabajo se presenta un avance del estado del proyecto, así como los principales inconvenientes encontrados.

\section{Desarrollo}

Como se mencionó anteriormente, la adquisición de datos se realizó con un dispositivo Structure mediante el cual es capaz de capturar un modelo tridimensional en forma de mesh o maya, en el cual está contenida tanto la geometría del objetivo como de todo lo que se encuentre en las cercanías del mismo.

Las mayas obtenidas de esta manera cuentan con una cantidad de puntos muy variables, dependientes de varios factores, siendo uno de los más importantes la cantidad de superficie no perteneciente al animal que se captura. A este respecto, es necesario aclarar que resulta difícil limitar el área capturada por el sensor, ya que para una medición precisa se necesita captar la mayor cantidad de superficie del animal posible, para lo cual es necesario rodear el mismo completamente realizando cambios de angulo del dispositivo, lo cual lleva a su vez a que elementos del entorno sean también capturados en el proceso. Entre los elementos del ambiente, es común encontrar no solo elementos de suelo sino también arbustos y otros animales dado que en ocasiones los individuos suelen tomar descansos en espacios con vegetación.

En general, el proceso completo de adquisición de datos de un solo animal se hace de la siguiente manera: en primera instancia se debe seleccionar el animal a escanear, el cual por limitaciones del sensor debe estar en un lugar a la sombra o sin incidencia directa del sol. Dado que no se utiliza ningún tipo de tranquilizante, una única persona se acerca y toma la captura del animal. Para disminuir la posibilidad de que el sonido producido por los pasos despierte al animal, se monta el dispositivo iPad en una extensión para lograr un alcance del 
cuerpo completo limitando al mínimo el movimiento del usuario alrededor del objetivo. En caso de que el animal se mueva por cualquier motivo, es necesario volver a tomar la captura o, en caso de no ser posible, buscar una manera de eliminar el ruido producido en la maya por dicha acción.

Si bien la captura de los modelos con el sensor en óptimas condiciones puede ser rápida, no resulta así la captura física de los mismos para tomar mediciones contra las que comparar, razón por la cual no es posible contar con una gran cantidad de datos. A este respecto se debe tener en cuenta que los animales no solo se encuentran muy alejados de centros urbanos, sino que por sus dimensiones, es necesario contar con herramientas especializadas, un equipo de trabajo de varias personas y mantener los mismos por varios días, lo cual hace que cada etapa de recolección de datos sea muy compleja y costosa (tanto en tiempo como en dinero).
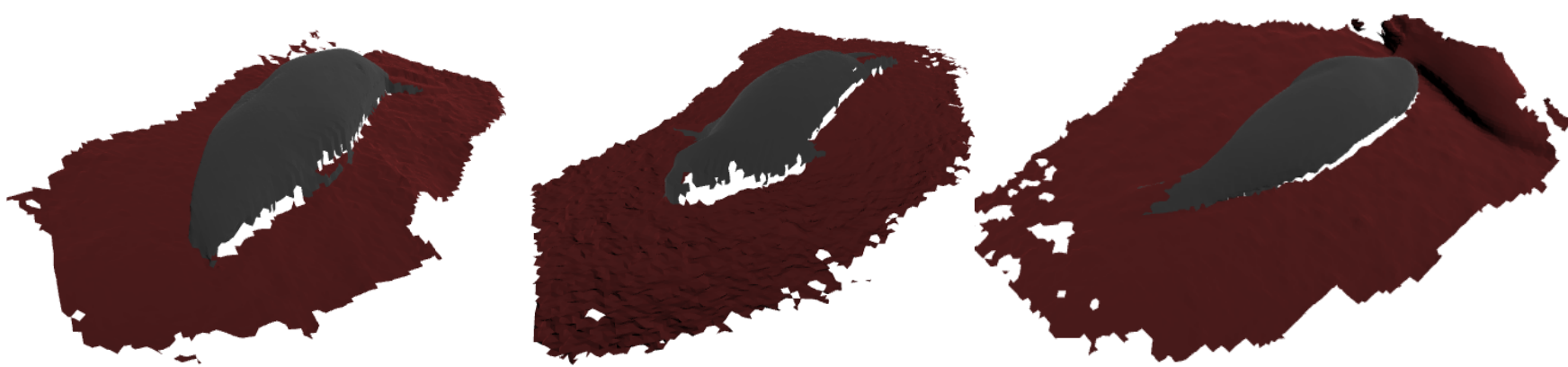

Figura 1: Ejemplo de mayas escaneadas. Las secciones de color rojizo son las detectadas como no pertenecientes al cuerpo del animal, mientras que las blancas son lugares en los que el sensor no pudo registrar puntos. Notar el cuerpo extraño en la imagen de la derecha, y cómo se identifica el mismo como ajena al animal .

En última instancia, los programas disponibles para la utilización del sensor son de uso general, por lo cual la maya resultante no hace ningún tipo de distinción entre las partes que corresponde al cuerpo del animal y las que no, razón por la cual es necesario realizar una limpieza de las mismas. A este respecto, la manera que mostró ser más efectiva para eliminar suelo es mediante una detección sencilla realizada a través de una estimación del mejor plano, filtrando los mismos acorde a las normales de cada punto. En general, este procedimiento muestra ser bastante efectivo eliminando de la maya la mayoría de las zonas que no corresponden al animal aún cuando el suelo no sea del todo regular ni plano (ver Figura 1).

En el caso de mayas cuyos modelos presentan ruido generado por movimientos hechos por el animal durante la tarea de escaneado, las deformaciones resultan difíciles de eliminar de manera automática (se puede ver un ejemplo en la Figura 2). Para hacer frente a este problema se han probado varios tipos de filtros, así como también varias arquitecturas de redes neuronales. Si bien algunas de estas últimas ya han sido bien probadas para la segmentación de nubes de puntos (tales como Badrinarayanan(2017) , Qi(2017) y Landrieu (2018) ), las mismas resultan demasiado complejas para el objetivo de este trabajo, lo cual se hace evidente en relación a la cantidad de datos que se normalmente se requiere para entrenar este tipo de algoritmos.

Actualmente el equipo está investigando, diseñando y evaluando algoritmos y arquitecturas mucho más simples a fin de conseguir una solución más adecuada para este proyecto (una posibilidad fue presentada en Almonacid (2018) ).

En cuanto a las redes neuronales, es necesario realizar un muestreo tomando cantidades fijas de puntos de la superficie de los modelos (actualmente 1024), ya que las arquitecturas en estudio aún no admiten el ingreso de datos con cantidades variables de puntos. Esta limitación influye en la precisión del modelo resultante ya que para mayor cantidad de puntos, el procesamiento necesario se incrementa de manera considerable, aunque para el caso de estudio este nivel de precisión no presenta mayores inconvenientes.

Como siguiente etapa, se completa el modelo utilizando una reconstrucción de poisson ( Kazhdan (2006) ), aunque se están probando otros algoritmos para comparar resultados. A partir de esta reconstrucciones calcula el volumen (Fig. 3) y a posterior, la estimación de peso del animal. En la actualidad se cuenta con datos de volumen, pero aún no se ha calibrado el sistema para una adecuada estimación del peso de los mismos (razón por la cual no se presentan comparaciones del resultado final). 


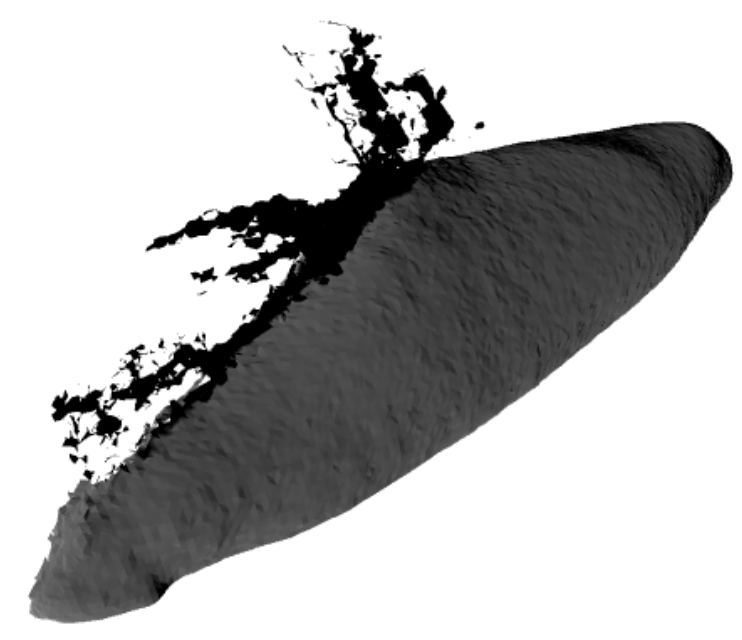

Figura 2: Modelo de un animal con ruido producido por movimiento de la aleta pectoral durante la tarea de escaneado.
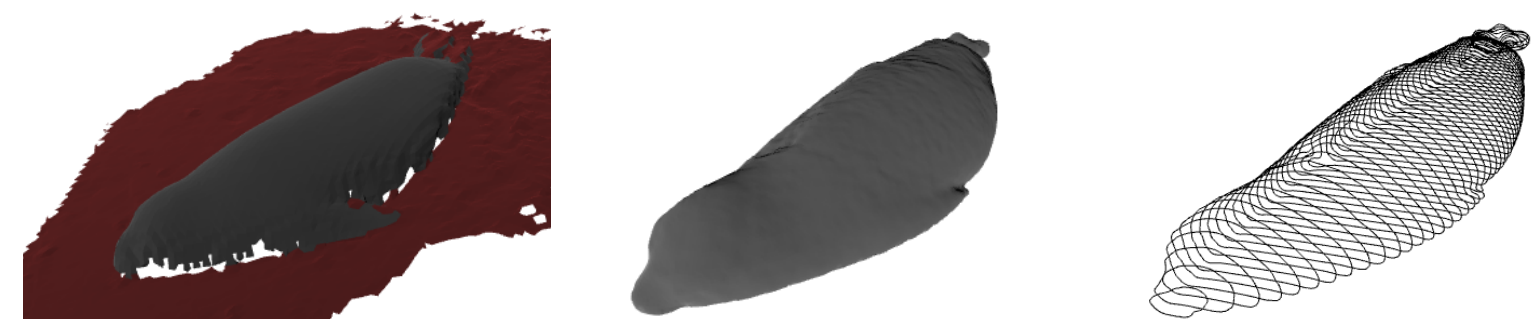

Figura 3: Diferentes estados de modelos para el cálculo del volumen. A la izquierda se ve un modelo segmentado, en el centro una reconstrucción de poisson, y a la derecha cortes transversales hechos sobre el último modelo (el cálculo de volumen a la manera tradicional puede ser simulado usando entre dos y cuatro de estas secciones y el largo).

\section{Conclusión}

A través de las pruebas realizadas se puede ver que la utilización de tecnologías de escaneo 3D para este tipo de tareas presentan grandes ventajas, con repercusiones tanto en aspectos de calidad de datos como en cuanto a seguridad y coste de obtención de los mismos.

En cuanto al análisis de los datos, uno de los problemas encontrados radica en la dificultad que presenta el consumo y procesamiento de nubes de puntos de manera nativa y/o transparente. Si bien en los últimos años se han presentado grandes avances para el dominio 3D (en particular para el análisis de nubes de puntos), es necesario continuar con estudios que permitan un procesamiento rápido y computacionalmente menos costoso, así como también más transparente de este tipo de datos.

Del mismo modo, en este momento el grupo sigue trabajando para aplicar otros modelos muy prometedores para abordar dichas dificultades, tales como los presentados en Hermosilla (2018) en cuanto al análisis, y en Hanocka (2020) para la reconstrucción.

\section{Referencias}

Bell, C. M. (1997). Growth of the southern elephant seal Mirounga leonina (Linnaeus 1758) at Macquarie Island (Doctoral dissertation, University of Tasmania). 
Landrieu, L., \& Simonovsky, M. (2018). Large-scale point cloud semantic segmentation with superpoint graphs. In Proceedings of the IEEE Conference on Computer Vision and Pattern Recognition (pp. 45584567)..

Badrinarayanan, V., Kendall, A., \& Cipolla, R. (2017). Segnet: A deep convolutional encoder-decoder architecture for image segmentation. IEEE transactions on pattern analysis and machine intelligence, 39(12), 2481-2495.

Qi, C. R., Su, H., Mo, K., \& Guibas, L. J. (2017). Pointnet: Deep learning on point sets for 3d classification and segmentation. In Proceedings of the IEEE conference on computer vision and pattern recognition (pp. 652-660).

Almonacid, Jonathan \& Cintas, Celia \& Derieux, Claudio \& Lewis, Mirtha. (2018). Point Cloud Denoising using Deep Learning. 1-5. 10.1109/CACIDI.2018.8584185.

Kazhdan, M., Bolitho, M., \& Hoppe, H. (2006, June). Poisson surface reconstruction. In Proceedings of the fourth Eurographics symposium on Geometry processing (Vol. 7).

Occipital (2020, march 6). What are the technical specifications of Structure Sensor (ST01)?. https://support.structure.io/article/157-what-are-the-structure-sensors-technical-specifications

Hermosilla, P., Ritschel, T., Vázquez, P. P., Vinacua, À., \& Ropinski, T. (2018). Monte carlo convolution for learning on non-uniformly sampled point clouds. ACM Transactions on Graphics (TOG), 37(6), 1-12.

Hanocka, R., Metzer, G., Giryes, R., \& Cohen-Or, D. (2020). Point2Mesh: A Self-Prior for Deformable Meshes. arXiv preprint arXiv:2005.11084. 